\title{
Effects of time-varying $\beta$ in SNLS3 on constraining interacting dark energy models
}

\author{
Shuang Wang ${ }^{1}$, Yong-Zhen Wang ${ }^{1}$, Jia-Jia Geng ${ }^{1}$, Xin Zhang ${ }^{1,2, a}$ \\ ${ }^{1}$ Department of Physics, College of Sciences, Northeastern University, Shenyang 110004, China \\ ${ }^{2}$ Center for High Energy Physics, Peking University, Beijing 100080, China
}

Received: 2 June 2014 / Accepted: 21 October 2014 / Published online: 11 November 2014

(C) The Author(s) 2014. This article is published with open access at Springerlink.com

\begin{abstract}
It has been found that, for the Supernova Legacy Survey three-year (SNLS3) data, there is strong evidence for the redshift evolution of the color-luminosity parameter $\beta$. In this paper, adopting the $w$-cold-dark-matter $(w \mathrm{CDM})$ model and considering its interacting extensions (with three kinds of interaction between dark sectors), we explore the evolution of $\beta$ and its effects on parameter estimation. In addition to the SNLS3 data, we also use the latest Planck distance priors data, the galaxy clustering data extracted from sloan digital sky survey data release 7 and baryon oscillation spectroscopic survey, as well as the direct measurement of Hubble constant $H_{0}$ from the Hubble Space Telescope observation. We find that, for all the interacting dark energy (IDE) models, adding a parameter of $\beta$ can reduce $\chi^{2}$ by $\sim 34$, indicating that a constant $\beta$ is ruled out at $5.8 \sigma$ confidence level. Furthermore, it is found that varying $\beta$ can significantly change the fitting results of various cosmological parameters: for all the dark energy models considered in this paper, varying $\beta$ yields a larger fractional CDM densities $\Omega_{c 0}$ and a larger equation of state $w$; on the other side, varying $\beta$ yields a smaller reduced Hubble constant $h$ for the $w$ CDM model, but it has no impact on $h$ for the three IDE models. This implies that there is a degeneracy between $h$ and coupling parameter $\gamma$. Our work shows that the evolution of $\beta$ is insensitive to the interaction between dark sectors, and then highlights the importance of considering $\beta$ 's evolution in the cosmology fits.
\end{abstract}

\section{Introduction}

Cosmic acceleration is one of the biggest puzzles in modern cosmology [1-17]. There are mainly two approaches to explain this extremely counterintuitive phenomenon: dark energy (DE) [18-88] and modified gravity (MG) [89-98]. For recent reviews, see [99-108].

\footnotetext{
a e-mail: zhangxin@mail.neu.edu.cn
}

Cosmological observations are of essential importance to understanding cosmic acceleration, and one of the most important observations is type Ia supernovae (SNe Ia) [109113]. In 2010, the Supernova Legacy Survey (SNLS) group released their 3 years data, i.e. SNLS3 dataset [114]. Soon after, using this dataset, Conley et al. [115] and Sullivan et al. [116] presented the $\mathrm{SN}$-only cosmological results and the joint cosmological constraints, respectively. Unlike other supernova (SN) group, the SNLS team treated two important quantities, stretch-luminosity parameter $\alpha$ and colorluminosity parameter $\beta$ of SNe Ia, as free model parameters.

There are many factors that can lead to systematic uncertainties of SNe Ia. One of the most important factors is the potential SN evolution, i.e. the possibility for the redshift evolution of $\alpha$ and $\beta$. The current studies show that $\alpha$ is consistent with a constant, but the hints for the evolution of $\beta$ have been found in [117-121]. For example, in [122], using a linear $\beta(z)=\beta_{0}+\beta_{1} z$, Mohlabeng and Ralston studied the case of Union2.1 dataset and found that $\beta$ deviates from a constant at $7 \sigma$ confidence levels (CL). Wang and Wang [123] found, for the SNLS3 data, $\beta$ increases significantly with $z$ at the $6 \sigma \mathrm{CL}$; moreover, they proved that this conclusion is insensitive to the lightcurve fitter models, or the functional form of $\beta(z)$ assumed [123]. Therefore, the evolution of $\beta$ is a common phenomenon for various SN datasets, and should be taken into account seriously.

It is very interesting to study the effects of a time-varying $\beta$ on parameter estimation. Wang et al. [124] explored this issue by considering the $\Lambda$-cold-dark-matter $(\Lambda \mathrm{CDM})$ model, the $w \mathrm{CDM}$ model, and the Chevallier-Polarski-Linder (CPL) model. Soon after, Wang et al. [125] studied the case of holographic dark energy (HDE) model, which is a physically plausible DE candidate based on the holographic principle. It is found that, for all these DE models, adding a parameter of $\beta$ can reduce $\chi_{\min }^{2}$ by $\sim 36$; in addition, considering the evolution of $\beta$ is helpful in reducing the tension between $\mathrm{SN}$ and other cosmological observations. It should be mentioned 
that, in principle, there is always an important possibility that DE directly interacts with CDM. This factor was not considered in $[124,125]$. To do a comprehensive analysis on the effects of a time-varying $\beta$, it is necessary to extend the corresponding discussions to the case of interacting dark energy (IDE) models.

In this paper, we explore the effects of a time-varying $\beta$ on the cosmological constraints of the IDE model. Three kinds of interaction terms are taken into account. In addition to the SNLS3 data, we also use the Planck distance prior data [126], the galaxy clustering (GC) data from sloan digital sky survey (SDSS) data release 7 (DR7) [127] and baryon oscillation spectroscopic survey (BOSS) [128], as well as the direct measurement of Hubble constant $H_{0}=73.8 \pm 2.4 \mathrm{~km} / \mathrm{s} / \mathrm{Mpc}$ from the Hubble Space Telescope (HST) observation [17].

We describe our method in Sect. 2, present our results in Sect. 3, and conclude in Sect. 4. In this paper, we assume today's scale factor $a_{0}=1$, thus the redshift $z=a^{-1}-1$. The subscript " 0 " always indicates the present value of the corresponding quantity, and the natural units are used.

\section{Methodology}

\subsection{Theoretical models}

In this paper, we consider a non-flat universe. The Friedmann equation can be written as

$3 M_{\mathrm{pl}}^{2} H^{2}=\rho_{c}+\rho_{d e}+\rho_{r}+\rho_{b}+\rho_{k}$,

where $M_{\mathrm{pl}} \equiv 1 / \sqrt{8 \pi G}$ is the reduced Planck mass, $\rho_{c}, \rho_{d e}$, $\rho_{r}, \rho_{b}$ and $\rho_{k}$ are the energy densities of CDM, DE, radiation, baryon and curvature, respectively. The reduced Hubble parameter $E(z) \equiv H(z) / H_{0}$ satisfies

$$
\begin{aligned}
E^{2}= & \Omega_{c 0} \frac{\rho_{c}}{\rho_{c 0}}+\Omega_{d e 0} \frac{\rho_{d e}}{\rho_{d e 0}}+\Omega_{r 0} \frac{\rho_{r}}{\rho_{r 0}}+\Omega_{b 0} \frac{\rho_{b}}{\rho_{b 0}} \\
& +\Omega_{k 0} \frac{\rho_{k}}{\rho_{k 0}},
\end{aligned}
$$

where $\Omega_{c 0}, \Omega_{d e 0}, \Omega_{r 0}, \Omega_{b 0}$ and $\Omega_{k 0}$ are the present fractional densities of CDM, DE, radiation, baryon and curvature, respectively. Since $\Omega_{c 0}+\Omega_{d e 0}+\Omega_{r 0}+\Omega_{b 0}+\Omega_{k 0}=1$, we do not treat $\Omega_{d e 0}$ as an independent parameter in this paper. In addition, $\rho_{r}=\rho_{r 0}(1+z)^{4}, \rho_{b}=\rho_{b 0}(1+z)^{3}$, $\rho_{k}=\rho_{k 0}(1+z)^{2}, \Omega_{r 0}=\Omega_{m 0} /\left(1+z_{\mathrm{eq}}\right)$, where $\Omega_{m 0}=$ $\Omega_{c 0}+\Omega_{b 0}$ and $z_{\mathrm{eq}}=2.5 \times 10^{4} \Omega_{m 0} h^{2}\left(T_{\mathrm{cmb}} / 2.7 \mathrm{~K}\right)^{-4}$ (here we take $T_{\mathrm{cmb}}=2.7255 \mathrm{~K}$ ).

Considering the interaction between dark sectors, the dynamical evolutions of CDM and DE become

$\dot{\rho}_{c}+3 H \rho_{c}=Q$,

$\dot{\rho}_{d e}+3 H\left(\rho_{d e}+p_{d e}\right)=-Q$, where the over dot denotes the derivative with respect to the cosmic time $t, p_{d e}=w \rho_{d e}$ is the pressure of $\mathrm{DE}, w$ is the equation of state of $\mathrm{DE}$, and $Q$ is the interaction term, which describes the energy transfer rate between CDM and DE. Notice that $a=\frac{1}{1+z}$ and $H=\frac{\dot{a}}{a}$, we have $\frac{\mathrm{d}}{\mathrm{d} t}=-H(1+z) \frac{\mathrm{d}}{\mathrm{d} z}$. Then Eqs. (3) and (4) can be rewritten as

$$
\begin{aligned}
& (1+z) \frac{\mathrm{d} \rho_{c}}{\mathrm{~d} z}-3 \rho_{c}=-Q / H, \\
& (1+z) \frac{\mathrm{d} \rho_{d e}}{\mathrm{~d} z}-3(1+w) \rho_{\mathrm{d} e}=Q / H .
\end{aligned}
$$

The solutions of these two equations depend on the specific forms of $Q$.

So far, the microscopic origin of interaction between dark sectors is still a big puzzle to us. To study the issue of interaction, one has to write down the possible forms of $Q$ by hand. In this paper we consider the following four cases:

$$
\begin{aligned}
& Q_{0}=0, \\
& Q_{1}=3 \gamma H \rho_{c}, \\
& Q_{2}=3 \gamma H \rho_{d e}, \\
& Q_{3}=3 \gamma H \frac{\rho_{c} \rho_{d e}}{\rho_{c}+\rho_{d e}},
\end{aligned}
$$

where $\gamma$ is a dimensionless coupling parameter describing the strength of interaction. Notice that the model with $Q_{0}$ denotes the case without dark sector interaction; the models with $Q_{1}$ and $Q_{2}$ are very popular, and both of them have been widely studied in the literature (see, e.g., [129-153]); the model with $Q_{3}$ is proposed in [154], and it can solve the early-time superhorizon instability and future unphysical CDM density problems at the same time. For simplicity, hereafter we call them $w \mathrm{CDM}$ model, I $w \mathrm{CDM} 1$ model, I $w$ CDM2 model, and I $w$ CDM3 model, respectively.

For the $w \mathrm{CDM}$ model, the reduced Hubble parameter $E(z) \equiv H(z) / H_{0}$ can be written as

$$
\begin{gathered}
E(z)=\left(\Omega_{r 0}(1+z)^{4}+\left(\Omega_{c 0}+\Omega_{b 0}\right)(1+z)^{3}+\Omega_{k 0}(1+z)^{2}\right. \\
\left.+\Omega_{d e 0}(1+z)^{3(1+w)}\right)^{1 / 2} .
\end{gathered}
$$

For the I $w$ CDM1 model, Eq. (5) has a general solution

$\rho_{c}=\rho_{c 0}(1+z)^{3(1-\gamma)}$.

Substituting Eq. (12) into Eq. (6) and using the initial condition $\rho_{d e}(z=0)=\rho_{d e 0}$, we get

$$
\begin{aligned}
\rho_{d e}= & \frac{\gamma \rho_{c 0}}{\gamma+w}\left((1+z)^{3(1+w)}-(1+z)^{3(1-\gamma)}\right) \\
& +\rho_{d e 0}(1+z)^{3(1+w)} .
\end{aligned}
$$


Then substituting Eqs. (12) and (13) into Eq. (2), we obtain

$$
\begin{aligned}
E(z)= & \left(\Omega_{r 0}(1+z)^{4}+\Omega_{b 0}(1+z)^{3}+\Omega_{k 0}(1+z)^{2}\right. \\
& +\Omega_{d e 0}(1+z)^{3(1+w)}+\Omega_{c 0}\left(\frac{\gamma}{w+\gamma}(1+z)^{3(1+w)}\right. \\
& \left.\left.+\frac{w}{w+\gamma}(1+z)^{3(1-\gamma)}\right)\right)^{1 / 2} .
\end{aligned}
$$

For the I $w$ CDM2 model, Eq. (6) has a general solution

$\rho_{d e}=\rho_{d e 0}(1+z)^{3(1+w+\gamma)}$.

Substituting Eq. (15) into Eq. (5) and using the initial condition $\rho_{c}(z=0)=\rho_{c 0}$, we get

$$
\begin{aligned}
\rho_{c}= & \rho_{c 0}(1+z)^{3}+\frac{\gamma \rho_{d e 0}}{w+\gamma}(1+z)^{3} \\
& -\frac{\gamma \rho_{d e 0}}{w+\gamma}(1+z)^{3(1+w+\gamma)} .
\end{aligned}
$$

Then substituting Eqs. (15) and (16) into Eq. (2), we have

$$
\begin{gathered}
E(z)=\left(\Omega_{r 0}(1+z)^{4}+\left(\Omega_{c 0}+\Omega_{b 0}\right)(1+z)^{3}+\Omega_{k 0}(1+z)^{2}\right. \\
\left.+\Omega_{d e 0}\left(\frac{\gamma}{w+\gamma}(1+z)^{3}+\frac{w}{w+\gamma}(1+z)^{3(1+w+\gamma)}\right)\right)^{1 / 2} .
\end{gathered}
$$

For the I $w$ CDM3 model, the energy densities of CDM and DE satisfy

$$
\begin{aligned}
\rho_{c}= & \rho_{c 0}(1+z)^{3} \\
& \times\left(\frac{\rho_{c 0}}{\rho_{c 0}+\rho_{d e 0}}+\frac{\rho_{d e 0}}{\rho_{c 0}+\rho_{d e 0}}(1+z)^{3(w+\gamma)}\right)^{-\frac{\gamma}{w+\gamma}},
\end{aligned}
$$

$$
\begin{aligned}
\rho_{d e}= & \rho_{d e 0}(1+z)^{3(1+w+\gamma)} \\
& \times\left(\frac{\rho_{c 0}}{\rho_{c 0}+\rho_{d e 0}}+\frac{\rho_{d e 0}}{\rho_{c 0}+\rho_{d e 0}}(1+z)^{3(w+\gamma)}\right)^{-\frac{\gamma}{w+\gamma}} .
\end{aligned}
$$

Substituting Eqs. (18) and (19) into Eq. (2), we get

$$
\begin{aligned}
E(z)= & \left(\Omega_{c 0} C(z)(1+z)^{3}+\Omega_{d e 0} C(z)(1+z)^{3(1+w+\gamma)}\right. \\
& \left.+\Omega_{r 0}(1+z)^{4}+\Omega_{b 0}(1+z)^{3}+\Omega_{k 0}(1+z)^{2}\right)^{1 / 2}
\end{aligned}
$$

where

$$
C(z)=\left(\frac{\Omega_{c 0}}{\Omega_{c 0}+\Omega_{d e 0}}+\frac{\Omega_{d e 0}}{\Omega_{c 0}+\Omega_{d e 0}}(1+z)^{3(w+\gamma)}\right)^{-\frac{\gamma}{w+\gamma}} .
$$

Note that in Eqs. (11), (14), (17), (20), and (21), $\Omega_{d e 0}$ is not an independent parameter, which is given by $\Omega_{d e 0}=$ $1-\Omega_{c 0}-\Omega_{b 0}-\Omega_{r 0}-\Omega_{k 0}$.

\subsection{Observational data}

In this subsection, we introduce how to include the SNLS3 data into the $\chi^{2}$ analysis.

For the SNLS3 sample, the observable is $m_{B}$, which is the rest-frame peak B-band magnitude of the SN. By considering three functional forms (linear case, quadratic case, and step function case), Wang and Wang [123] showed that the evolutions of $\alpha$ and $\beta$ are insensitive to functional form of $\alpha$ and $\beta$ assumed. So in this paper, we just adopt a constant $\alpha$ and a linear $\beta(z)=\beta_{0}+\beta_{1} z$. Then the predicted magnitude of an $\mathrm{SN}$ becomes

$m_{\text {mod }}=5 \log _{10} \mathcal{D}_{L}(z)-\alpha(s-1)+\beta(z) \mathcal{C}+\mathcal{M}$,

where $s$ and $\mathcal{C}$ are the stretch measure and the color measure for the SN light curve. Here $\mathcal{M}$ is a parameter representing some combination of SN absolute magnitude $M$ and Hubble constant $H_{0}$. It must be emphasized that, to include hostgalaxy information in the cosmological fits, Conley et al. [115] split the SNLS3 sample based on host-galaxy stellar mass at $10^{10} M_{\odot}$, and made $\mathcal{M}$ to be different for the two samples. Therefore, unlike other $\mathrm{SN}$ samples, there are two values of $\mathcal{M}, \mathcal{M}_{1}$ and $\mathcal{M}_{2}$, for the SNLS3 data Moreover, Conley et al. removed $\mathcal{M}_{1}$ and $\mathcal{M}_{2}$ from cosmology fits by analytically marginalizing over them (for more details, see the appendix $\mathrm{C}$ of [115], as well as the the public code which is available at https://tspace.library.utoronto.ca/handle/1807/24512). In this paper, we just follow the recipe of [115]. The luminosity distance $\mathcal{D}_{L}(z)$ is defined as

$\mathcal{D}_{L}(z) \equiv H_{0}\left(1+z_{\text {hel }}\right) r(z)$,

where $z$ and $z_{\text {hel }}$ are the CMB rest frame and heliocentric redshifts of SN. In addition, the comoving distance $r(z)$ is given by

$r(z)=H_{0}^{-1}\left|\Omega_{k 0}\right|^{-1 / 2} \operatorname{sinn}\left(\left|\Omega_{k 0}\right|^{1 / 2} \Gamma(z)\right)$,

where $\Gamma(z)=\int_{0}^{z} \frac{d z^{\prime}}{E\left(z^{\prime}\right)}$, and $\operatorname{sinn}(x)=\sin (x), x, \sinh (x)$ for $\Omega_{k 0}<0, \Omega_{k 0}=0$, and $\Omega_{k 0}>0$, respectively.

For a set of $N$ SNe with correlated errors, the $\chi^{2}$ function is

$\chi_{S N}^{2}=\Delta \mathbf{m}^{T} \cdot \mathbf{C}^{-1} \cdot \Delta \mathbf{m}$, 
Table 1 Fitting results for various constant $\beta$ and linear $\beta(z)$ cases, where the $\mathrm{SNe}+\mathrm{CMB}+\mathrm{GC}+H_{0}$ data are used

\begin{tabular}{|c|c|c|c|c|c|c|c|c|}
\hline \multirow[t]{2}{*}{ Parameters } & \multicolumn{2}{|l|}{$w \mathrm{CDM}$} & \multicolumn{2}{|l|}{$\mathrm{I} w \mathrm{CDM} 1$} & \multicolumn{2}{|l|}{$\mathrm{I} w \mathrm{CDM} 2$} & \multicolumn{2}{|l|}{$\mathrm{I} w \mathrm{CDM} 3$} \\
\hline & Const $\beta$ & Linear $\beta(z)$ & Const $\beta$ & Linear $\beta(z)$ & Const $\beta$ & Linear $\beta(z)$ & Const $\beta$ & Linear $\beta(z)$ \\
\hline$\alpha$ & $1.444_{-0.115}^{+0.079}$ & $1.423_{-0.095}^{+0.087}$ & $1.424_{-0.094}^{+0.104}$ & $1.398_{-0.066}^{+0.110}$ & $1.427_{-0.097}^{+0.096}$ & $1.421_{-0.101}^{+0.084}$ & $1.445_{-0.115}^{+0.082}$ & $1.393_{-0.068}^{+0.121}$ \\
\hline$\beta_{0}$ & $3.251_{-0.098}^{+0.113}$ & $1.518_{-0.378}^{+0.326}$ & $3.272_{-0.116}^{+0.087}$ & $1.438_{-0.372}^{+0.367}$ & $3.275_{-0.112}^{+0.084}$ & $1.474_{-0.369}^{+0.308}$ & $3.248_{-0.084}^{+0.110}$ & $1.505_{-0.402}^{+0.292}$ \\
\hline$\beta_{1}$ & & $4.926_{-0.869}^{+1.011}$ & & $5.102_{-0.924}^{+0.988}$ & & $4.970_{-0.819}^{+1.015}$ & & $4.886_{-0.747}^{+1.191}$ \\
\hline$\Omega_{c 0}$ & $0.224_{-0.010}^{+0.010}$ & $0.231_{-0.009}^{+0.011}$ & $0.232_{-0.015}^{+0.012}$ & $0.244_{-0.013}^{+0.016}$ & $0.226_{-0.013}^{+0.012}$ & $0.238_{-0.012}^{+0.020}$ & $0.225_{-0.011}^{+0.014}$ & $0.244_{-0.016}^{+0.013}$ \\
\hline$\Omega_{b 0}$ & $0.042_{-0.002}^{+0.002}$ & $0.044_{-0.002}^{+0.002}$ & $0.041_{-0.002}^{+0.003}$ & $0.040_{-0.002}^{+0.003}$ & $0.041_{-0.002}^{+0.003}$ & $0.041_{-0.002}^{+0.003}$ & $0.042_{-0.002}^{+0.002}$ & $0.042_{-0.003}^{+0.002}$ \\
\hline$\Omega_{k 0}$ & $0.00046_{-0.003}^{+0.004}$ & $0.0032_{-0.0041}^{+0.0038}$ & $0.0039_{-0.0061}^{+0.0044}$ & $0.0095_{-0.0059}^{+0.0050}$ & $0.0061_{-0.0162}^{+0.0142}$ & $0.0192_{-0.0165}^{+0.0180}$ & $0.0046_{-0.0131}^{+0.0160}$ & $0.0194_{-0.0159}^{+0.0194}$ \\
\hline$\gamma$ & & & $-0.0028_{-0.0031}^{+0.0043}$ & $-0.0053_{-0.0026}^{+0.0035}$ & $-0.0105_{-0.0295}^{+0.0310}$ & $-0.0322_{-0.0396}^{+0.0300}$ & $-0.0198_{-0.0752}^{+0.0613}$ & $-0.0732_{-0.0823}^{+0.0684}$ \\
\hline$w$ & $-1.118_{-0.071}^{+0.065}$ & $-1.042_{-0.072}^{+0.068}$ & $-1.105_{-0.069}^{+0.075}$ & $-1.016_{-0.063}^{+0.075}$ & $-1.124_{-0.062}^{+0.070}$ & $-1.052_{-0.068}^{+0.070}$ & $-1.116_{-0.072}^{+0.059}$ & $-1.038_{-0.080}^{+0.068}$ \\
\hline$h$ & $0.725_{-0.014}^{+0.014}$ & $0.716_{-0.015}^{+0.014}$ & $0.739_{-0.023}^{+0.019}$ & $0.743_{-0.024}^{+0.016}$ & $0.734_{-0.025}^{+0.018}$ & $0.735_{-0.024}^{+0.017}$ & $0.732_{-0.021}^{+0.022}$ & $0.729_{-0.018}^{+0.027}$ \\
\hline$\chi_{\min }^{2}$ & 422.696 & 388.508 & 422.376 & 387.128 & 422.674 & 387.814 & 422.642 & 387.716 \\
\hline
\end{tabular}

where $\Delta m \equiv m_{B}-m_{\text {mod }}$ is a vector with $N$ components, and $\mathbf{C}$ is the $N \times N$ covariance matrix of the $\mathrm{SN}$, given by

$\mathbf{C}=\mathbf{D}_{\text {stat }}+\mathbf{C}_{\text {stat }}+\mathbf{C}_{\text {sys }}$

$\mathbf{D}_{\text {stat }}$ is the diagonal part of the statistical uncertainty, given by [115]

$$
\begin{aligned}
\mathbf{D}_{\text {stat }, i i}= & \sigma_{m_{B}, i}^{2}+\sigma_{\text {int }}^{2}+\sigma_{\text {lensing }}^{2}+\sigma_{\text {host correction }}^{2} \\
& +\left[\frac{5\left(1+z_{i}\right)}{z_{i}\left(1+z_{i} / 2\right) \ln 10}\right]^{2} \sigma_{z, i}^{2} \\
& +\alpha^{2} \sigma_{s, i}^{2}+\beta\left(z_{i}\right)^{2} \sigma_{\mathcal{C}, i}^{2}+2 \alpha C_{m_{B} s, i} \\
& -2 \beta\left(z_{i}\right) C_{m_{B}} \mathcal{C}, i \\
& -2 \alpha \beta\left(z_{i}\right) C_{s \mathcal{C}, i},
\end{aligned}
$$

where $C_{m_{B} s, i}, C_{m_{B}} \mathcal{C}, i$, and $C_{s \mathcal{C}, i}$ are the covariances between $m_{B}, s$, and $\mathcal{C}$ for the $i$ th $\mathrm{SN}, \beta_{i}=\beta\left(z_{i}\right)$ are the values of $\beta$ for the $i$ th $\mathrm{SN}$. Notice that $\sigma_{z, i}^{2}$ includes a peculiar velocity residual of 0.0005 (i.e., $150 \mathrm{~km} / \mathrm{s}$ ) added in quadrature. Following [115], we fix the intrinsic scatter $\sigma_{\text {int }}$ to ensure that $\chi^{2} /$ dof $=1$. Varying $\sigma_{\text {int }}$ could have a significant impact on parameter estimation; see [119,155] for details.

We define $\mathbf{V} \equiv \mathbf{C}_{\text {stat }}+\mathbf{C}_{\text {sys }}$, where $\mathbf{C}_{\text {stat }}$ and $\mathbf{C}_{\text {sys }}$ are the statistical and systematic covariance matrices, respectively. After treating $\beta$ as a function of $z, \mathbf{V}$ is given in the form,

$$
\begin{aligned}
\mathbf{V}_{i j}= & V_{0, i j}+\alpha^{2} V_{a, i j}+\beta_{i} \beta_{j} V_{b, i j}+\alpha V_{0 a, i j}+\alpha V_{0 a, j i} \\
& -\beta_{j} V_{0 b, i j}-\beta_{i} V_{0 b, j i}-\alpha \beta_{j} V_{a b, i j}-\alpha \beta_{i} V_{a b, j i} .
\end{aligned}
$$

It must be stressed that, while $V_{0}, V_{a}, V_{b}$, and $V_{0 a}$ are the same as the "normal" covariance matrices given by the SNLS data archive, $V_{0 b}$, and $V_{a b}$ are not the same as the ones given there. This is because the original matrices of SNLS3 are produced by assuming $\beta$ is constant. We have used the $V_{0 b}$ and $V_{a b}$

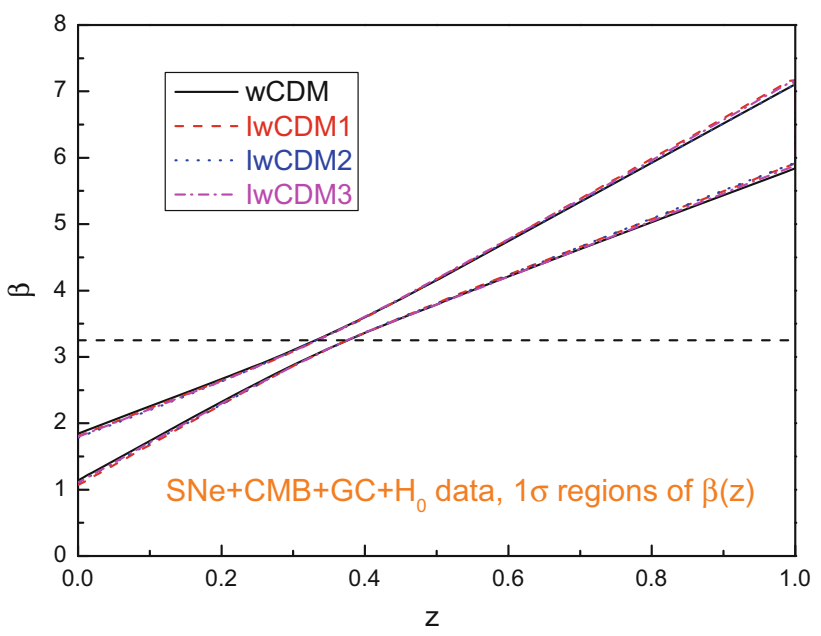

Fig. $11 \sigma$ confidence constraints for the evolution of $\beta(z)$, given by the $\mathrm{SNe}+\mathrm{CMB}+\mathrm{GC}+H_{0}$ data, for the $w \mathrm{CDM}$ model, the I $w \mathrm{CDM} 1$ model, the I $w \mathrm{CDM} 2$ model, and the I $w \mathrm{CDM} 3$ model. The solid black lines denote the $w \mathrm{CDM}$ model, the dashed red lines denote the I $w \mathrm{CDM} 1$ model, the dotted blue lines denote the I $w \mathrm{CDM} 2$ model, and the dasheddotted pink lines denote the I $w \mathrm{CDM} 3$ model. To make a comparison, for the $w \mathrm{CDM}$ model, the best-fit result of the constant $\beta$ case is also plotted, shown as the horizontal dashed black line

matrices for the "Combined" set that are applicable when varying $\beta(z)$ (A. Conley, private communication, 2013).

To improve the cosmological constraints, we also use some other cosmological observations, including the Planck distance prior data [126], the GC data extracted from SDSS DR7 [127] and BOSS [128], as well as the direct measurement of Hubble constant $H_{0}=73.8 \pm 2.4 \mathrm{~km} / \mathrm{s} / \mathrm{Mpc}$ from the HST observations [17]. For the details of including Planck and GC data into the $\chi^{2}$ analysis, see [124]. Now the total 

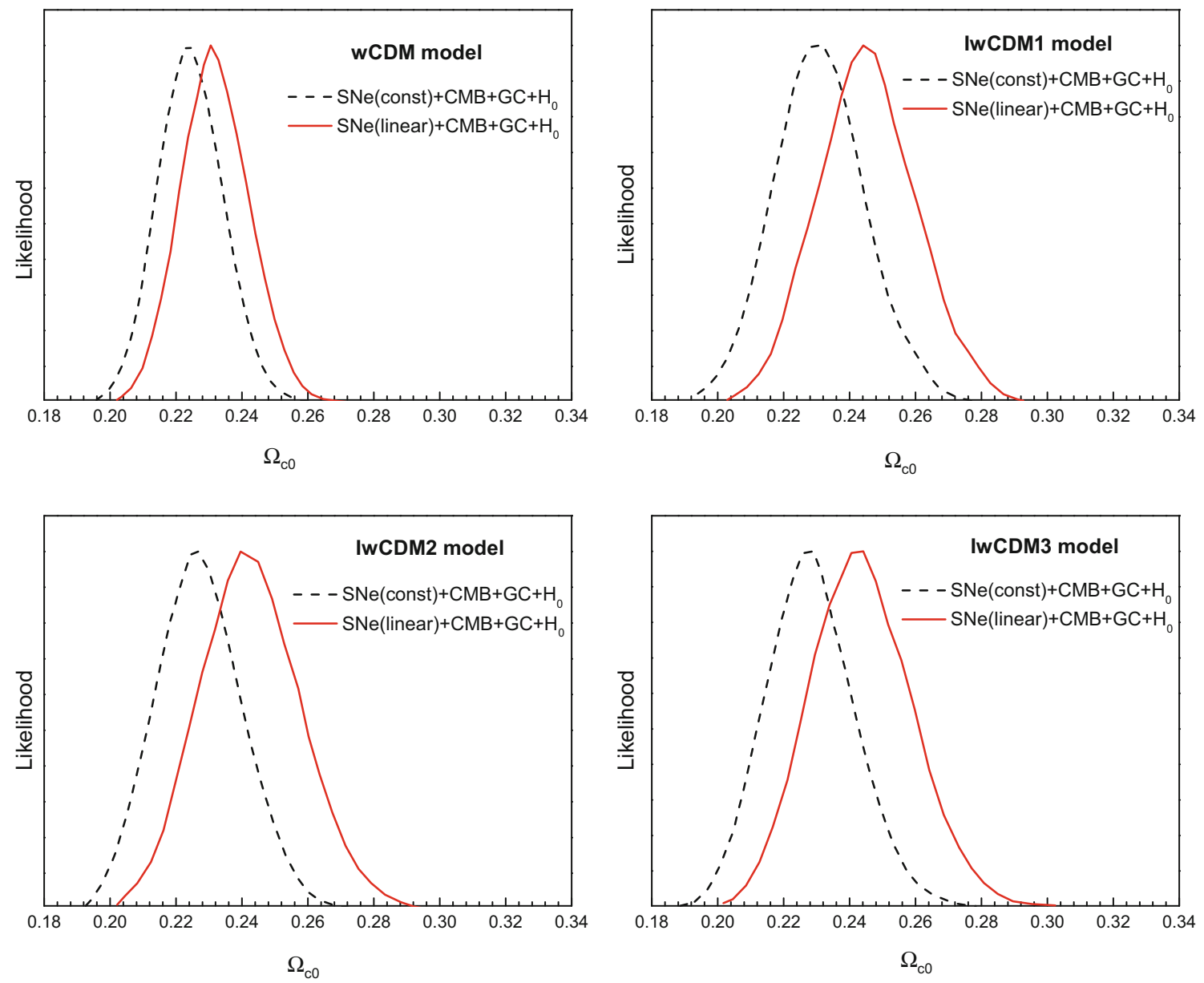

Fig. 2 The $1 \mathrm{D}$ marginalized probability distributions of $\Omega_{c 0}$, given by the $\mathrm{SNe}+\mathrm{CMB}+\mathrm{GC}+H_{0}$ data, for the $w \mathrm{CDM}$ model and the three IDE models. Both the results of the constant $\beta$ (dashed black lines) and the linear $\beta(z)$ (solid red lines) cases are presented

$\chi^{2}$ function is

$\chi^{2}=\chi_{\mathrm{SN}}^{2}+\chi_{\mathrm{CMB}}^{2}+\chi_{\mathrm{GC}}^{2}+\chi_{H_{0}}^{2}$.

In addition, assuming the measurement errors are Gaussian, the likelihood function satisfies

$\mathcal{L} \propto \mathrm{e}^{-\chi^{2} / 2}, \quad$ Likelihood $\equiv \mathcal{L} / \mathcal{L}_{\max }=\mathcal{L} / \mathcal{L}\left(\chi_{\text {min }}^{2}\right)$

It should be mentioned that in this paper we just use the purely geometric measurements, and do not consider the cosmological perturbations in the DE models. As analyzed in detail in [156], adopting a new framework for calculating the perturbations, the cosmological perturbations will always be stable in all IDE models (for a related discussion concerning the stability, see [154]). Therefore, the use of the Planck distance prior is sufficient for our purpose.

Finally, we perform an MCMC likelihood analysis [157] to obtain $\mathcal{O}\left(10^{6}\right)$ samples for each model considered in this paper.

\section{Results}

\subsection{Evolution of $\beta$}

In this subsection, we explore the evolution of $\beta$ in the frame of IDE.

In Table 1, we list the fitting results for various constant $\beta$ and linear $\beta(z)$ cases, where the $\mathrm{SNe}+\mathrm{CMB}+\mathrm{GC}+H_{0}$ data are used. An obvious feature of this table is that varying $\beta$ can significantly improve the fitting results: for all the models, adding a parameter of $\beta$ can reduce the best-fit values of $\chi^{2}$ by $\sim 34$. Based on the Wilk theorem, 34 units of $\chi^{2}$ is equivalent to a Gaussian fluctuation of $5.8 \sigma$. This means that the result of $\beta_{1}=0$ is ruled out at $5.8 \sigma$ confidence level (CL). As shown in [124,125], for the cases of various DE models (such as $\Lambda \mathrm{CDM}, w \mathrm{CDM}, \mathrm{CPL}$, and HDE model) without interaction, $\beta$ deviates from a constant at $6 \sigma \mathrm{CL}$. Therefore, we further confirm the redshift evolution of $\beta$ for the SNLS3 data.

In Fig. 1, using the $\mathrm{SNe}+\mathrm{CMB}+\mathrm{GC}+H_{0}$ data, we plot the $1 \sigma$ confidence constraints of $\beta(z)$, for the $w \mathrm{CDM}$ model, 

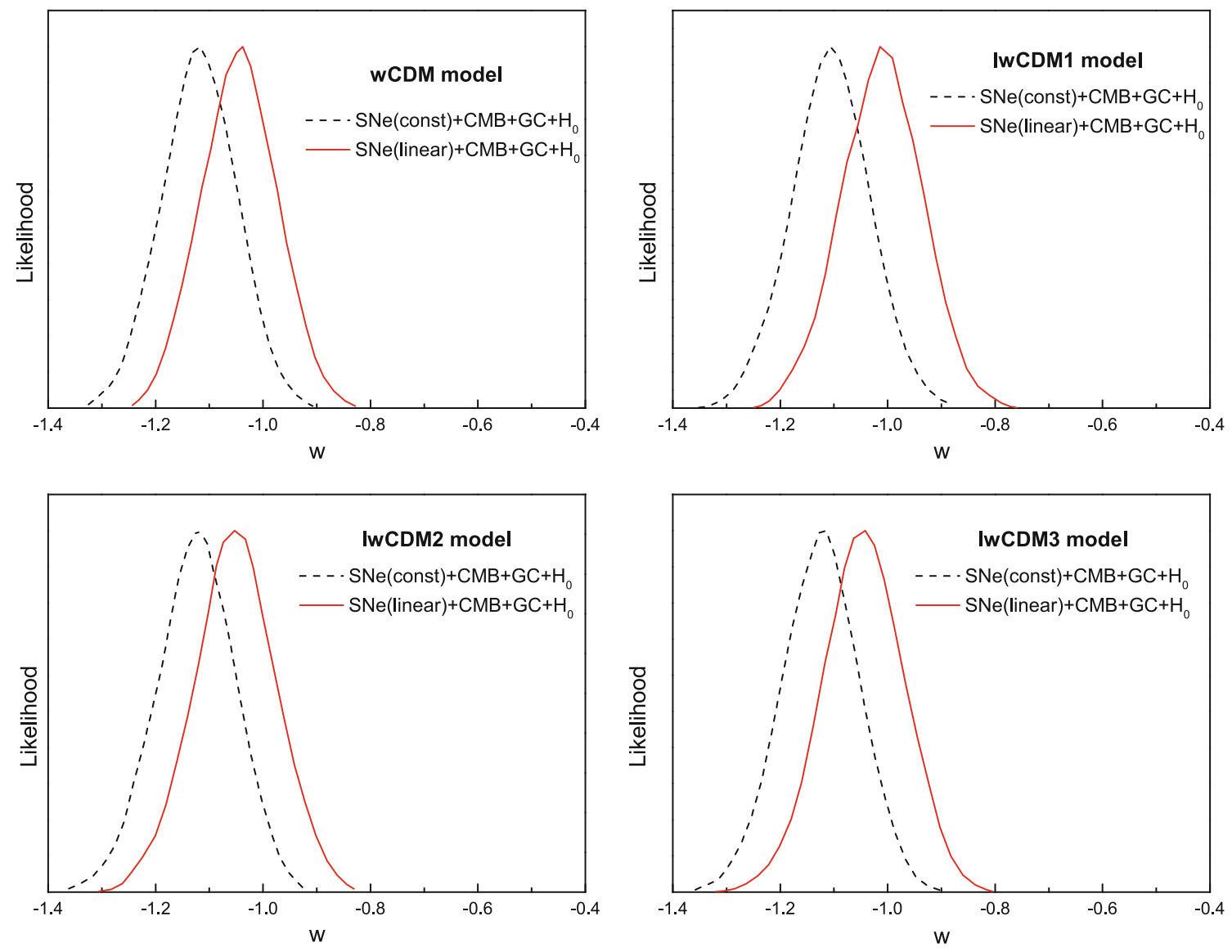

Fig. 3 The 1D marginalized probability distributions of $w$, given by the $\mathrm{SNe}+\mathrm{CMB}+\mathrm{GC}+H_{0}$ data, for the $w \mathrm{CDM}$ model and the three IDE models. Both the results of the constant $\beta$ (dashed black lines) and the linear $\beta(z)$ (solid red lines) cases are presented

the I $w$ CDM1 model, the I $w$ CDM2 model, and the I $w$ CDM3 model. For comparison, we also plot the best-fit result of the constant $\beta$ case for the $w \mathrm{CDM}$ model. From this figure one can see that the $1 \sigma$ regions of $\beta(z)$ of all these models are almost overlapping. This shows that the evolution of $\beta$ is independent of the IDE models. In addition, for all the models, $\beta(z)$ rapidly increases with $z$. This result is consistent with the results of $[124,125]$, showing that the evolution of $\beta$ is insensitive to dark energy models including those with interaction between dark sectors.

It should be pointed out that the evolutionary behaviors of $\beta(z)$ depends on the SN samples used. Mohlabeng and Ralston [122] found that, for the Union 2.1 SN data, $\beta(z)$ decreases with $z$. This is similar to the case of the PanSTARRS1 SN data [121].

It is interesting to study how different segments of the SNLS3 dataset give rise to different behavior of $\beta$. To do this, we perform the following test: (1) Per [115], we evenly divide the redshift range $[0,1]$ into 9 bins and assume that both $\alpha$ and $\beta$ are constant within each bin. (2) For each redshift bin, we make a small covariance matrix corresponding to only $\mathrm{SNe}$ in that bin. (3) Since we have already proved that the evolution of $\beta$ is insensitive to dark energy models, per
[115], we just adopt a fixed cosmological background (a flat $\Lambda \mathrm{CDM}$ model with $\Omega_{m 0}=0.26$ ) to do this test. (4) We fit $\alpha$ and $\beta$ separately for the 9 redshift bins. Based on the best-fit analysis, it is found that $\beta$ is relatively flat till the seventh bin, and then it rapidly increases along with redshift $z$. In other words, the rapid increase of $\beta(z)$ is mainly due to the contributions from high-redshift $(z>0.7) \mathrm{SN}$ samples of the SNLS3 dataset. It should be mentioned that, to keep this paper focused on its main purpose, here we just briefly present the conclusion, instead of describing all the detailed results of the test. To understand why high-redshift SNLS3 samples will yield this kind of evolutionary behavior of $\beta$, some numerical simulation studies may be needed. We will study this issue in future works.

\subsection{Effects of time-varying $\beta$}

In this subsection, we discuss the effects of varying $\beta$ on parameter estimation of IDE models.

In Fig. 2, using $\mathrm{SNe}+\mathrm{CMB}+\mathrm{GC}+\mathrm{H}_{0}$ data, we plot the 1D marginalized probability distributions of $\Omega_{c 0}$, for all the models considered in this paper. We find that varying $\beta$ yields a larger $\Omega_{c 0}$ : for the constant $\beta$ case, the best-fit results are 

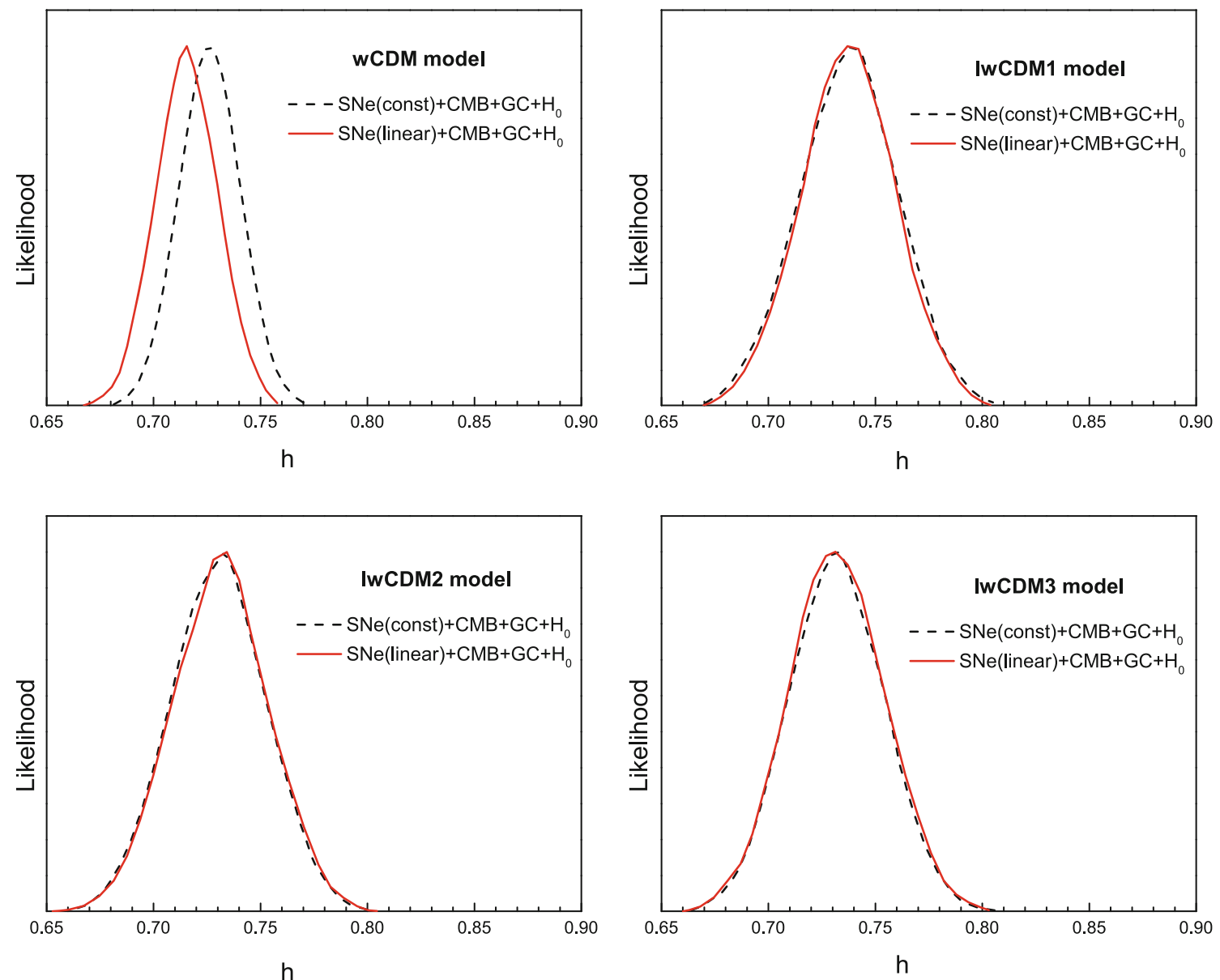

Fig. 4 The 1D marginalized probability distributions of $h$, given by the $\mathrm{SNe}+\mathrm{CMB}+\mathrm{GC}+H_{0}$ data, for the $w \mathrm{CDM}$ model and the three IDE models. Both the results of the constant $\beta$ (dashed black lines) and the linear $\beta(z)$ (solid red lines) cases are presented

$\Omega_{c 0}=0.224,0.232,0.226$, and 0.225 , for the $w \mathrm{CDM}$, the $\mathrm{I} w \mathrm{CDM} 1$, the I $w \mathrm{CDM} 2$, and the I $w \mathrm{CDM} 3$ model, respectively; while for the linear $\beta(z)$ case, the best-fit results are $\Omega_{c 0}=0.231,0.244,0.238$, and 0.244 , for the $w \mathrm{CDM}$, the $\mathrm{I} w \mathrm{CDM} 1$, the I $w \mathrm{CDM} 2$, and the I $w \mathrm{CDM} 3$ model, respectively. In addition, as shown in $[124,125]$, for various DE models without interaction term, a time-varying $\beta$ also yields a larger fractional matter density $\Omega_{m 0} \equiv \Omega_{c 0}+\Omega_{b 0}$. Therefore, we can conclude that the effects of varying $\beta$ on the present fractional matter density are insensitive to the interaction between dark sectors.

For all the models considered in this paper, the 1D marginalized probability distributions of $w$ are plotted in Fig. 3 . It is found that varying $\beta$ yields a larger $w$ : for the constant $\beta$ case, $w=-1.118_{-0.071}^{+0.065},-1.105_{-0.069}^{+0.075},-1.124_{-0.062}^{+0.070}$, and $-1.116_{-0.072}^{+0.059}$, for the $w \mathrm{CDM}$ model, the I $w \mathrm{CDM} 1$ model, the I $w \mathrm{CDM} 2$ model, and the I $w \mathrm{CDM} 3$ model, respectively; while for the linear $\beta(z)$ case, $w=-1.042_{-0.072}^{+0.068}$, $-1.016_{-0.063}^{+0.075},-1.052_{-0.068}^{+0.070}$, and $-1.038_{-0.080}^{+0.068}$, for the $w \mathrm{CDM}$ model, the I $w \mathrm{CDM} 1$ model, the I $w \mathrm{CDM} 2$ model, and the I $w \mathrm{CDM} 3$ model, respectively. In other words, $w<-1$ is preferred at more than $1 \sigma \mathrm{CL}$ for the constant $\beta$ case, while $w$ is consistent with -1 at $1 \sigma$ CL for the linear $\beta(z)$ case. This means that, compared to the constant $\beta$ case, the results from the varying $\beta$ case are in better agreement with a cosmological constant. This conclusion is consistent with the noninteracting cases $[124,125]$, showing that the effects of varying $\beta$ on $w$ are insensitive to the interaction between dark sectors.

In Fig. 4, we plot the 1D marginalized probability distributions of $h$, for all the models considered in this paper. It can be seen that, for the $w \mathrm{CDM}$ model, varying $\beta$ yields a smaller $h$; this result is consistent with the noninteracting cases $[124,125]$. However, for all the IDE models, the 1D distribution results of $h$ of the linear $\beta$ case are almost same with those of the constant $\beta$ case. In other words, once considering the interaction between dark sectors, varying $\beta$ will not change the fitting results of $h$. This result is quite different from the results of Figs. 2 and 3, showing that there is a degeneracy between $h$ and $\gamma$. 

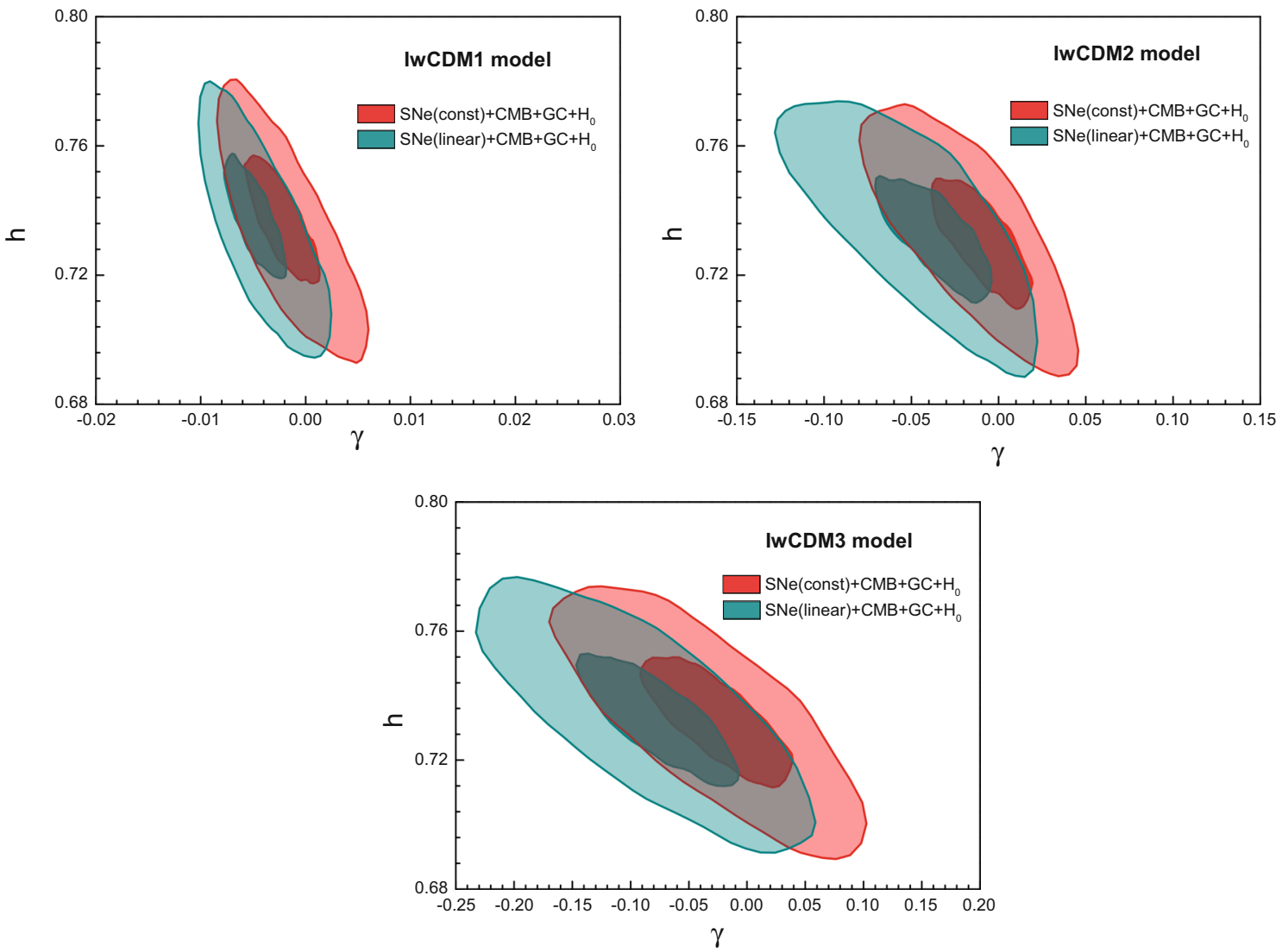

Fig. 5 The $1 \sigma$ and $2 \sigma$ confidence contours for $\{\gamma, h\}$, for the three IDE models. Both the results of the constant $\beta$ (red regions) and the linear $\beta(z)$ (dark cyan regions) cases are presented

Next, we turn to the constraints on interaction parameter $\gamma$. In Fig. 5, we plot $1 \sigma$ and $2 \sigma$ confidence contours for $\{\gamma, h\}$, for all the IDE models. Again, one can see that varying $\beta$ has no impact on $h$. To the contrary, varying $\beta$ yields a smaller $\gamma$ : for the constant $\beta$ case, the best-fit results are $\gamma=$ $-0.0028,-0.0105$, and -0.0198 , for the I $w$ CDM1 model, the I $w \mathrm{CDM} 2$ model, and the I $w \mathrm{CDM} 3$ model, respectively; while for the linear $\beta(z)$ case, the best-fit results are $\gamma=$ $-0.0053,-0.0322$, and -0.0732 , for the I $w$ CDM1 model, the I $w$ CDM 2 model, and the I $w$ CDM3 model, respectively. In other words, $\gamma<0$ is slightly more favored in the linear $\beta(z)$ case. This means that energy will transfer from dark matter to dark energy. In addition, we find that $\gamma$ and $h$ are anti-correlated, showing that there is a degeneracy between $h$ and $\gamma$.

In Fig. 6, to make a visual comparison among three interaction forms, we plot the $2 \sigma$ confidence contours for $\left\{\Omega_{c 0}, \gamma\right\}$, based on the linear $\beta(z)$ case, for all the IDE models. From this figure one can see that $\gamma$ is tightly constrained in the I $w \mathrm{CDM} 1$ model; to the contrary, $\gamma$ cannot be well constrained in the I $w \mathrm{CDM} 2$ and I $w \mathrm{CDM} 3$ models. This result is consistent with the result of [158], in which only the constant $\beta$ case was considered.

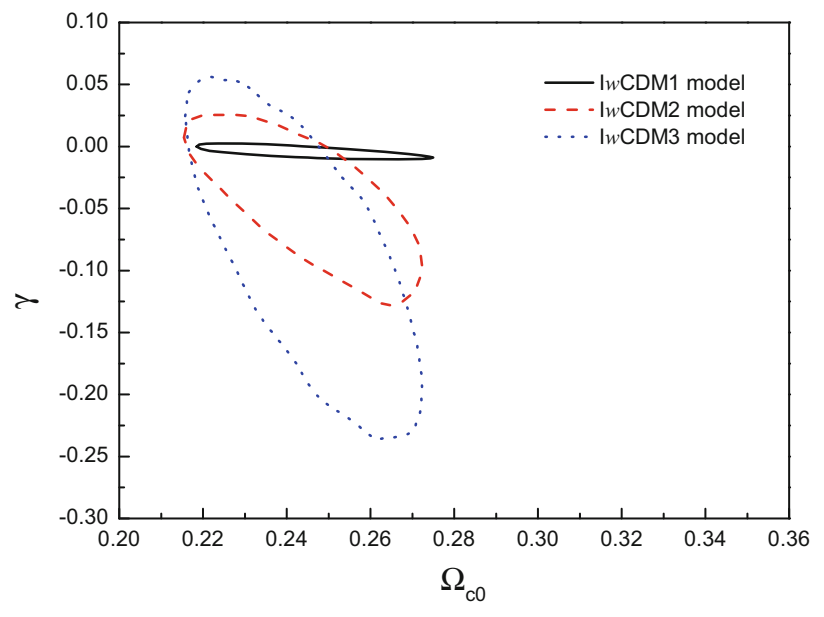

Fig. 6 The $2 \sigma$ confidence contours for $\left\{\Omega_{c 0}, \gamma\right\}$, based on the linear $\beta(z)$ case, for the I $w$ CDM1 model (solid black line), the I $w$ CDM2 model (dashed red line), and the IwCDM3 model (dotted blue line)

\section{Discussion and summary}

In recent years, more and more $\mathrm{SNe}$ Ia have been discovered, and the systematic errors of SNe Ia have drawn more 
and more attentions. One of the most important systematic uncertainties for SNe Ia is the potential SN evolution. The hints for the evolution of $\beta$ have been found [117-121]. For example, Mohlabeng and Ralston [122] studied the case of Union2.1 and found that $\beta$ deviates from a constant at $7 \sigma$ CL. Wang and Wang [123] found that, for the SNLS3 data, $\beta$ increases significantly with $z$ at the $6 \sigma \mathrm{CL}$; moreover, they proved that this conclusion is insensitive to the lightcurve fitter models, or the functional form of $\beta(z)$ assumed [123].

It is clear that a time-varying $\beta$ will have significant impact on parameter estimation. Adopting a constant $\alpha$ and a linear $\beta(z)=\beta_{0}+\beta_{1} z$, Wang et al. [124] explored this issue by considering the $\Lambda \mathrm{CDM}$ model, the $w \mathrm{CDM}$ model, and the CPL model. Soon after, Wang et al. [125] studied this issue in the frame of HDE model, which is a physically plausible DE candidate based on the holographic principle. It is found that, for all these DE models, $\beta$ deviates from a constant at $6 \sigma \mathrm{CL}$; in addition, considering the evolution of $\beta$ is helpful in reducing the tension between $\mathrm{SN}$ and other cosmological observations. It should be pointed out that, in principle, there is always an important possibility that DE directly interacts with CDM. This factor was not considered in $[124,125]$.

In this paper, we extend the corresponding discussions to the case of IDE model. To perform the cosmology fits, the $w \mathrm{CDM}$ model is adopted. Moreover, three kinds of interaction forms are considered: $Q_{1}=3 \gamma H \rho_{c}, Q_{2}=3 \gamma H \rho_{d e}$, and $Q_{3}=3 \gamma H \frac{\rho_{c} \rho_{d e}}{\rho_{c}+\rho_{d e}}$. In addition to the SNLS3 SN data, we also use the Planck distance priors data, the GC data extracted from SDSS DR7 and BOSS, as well as the direct measurement of Hubble constant from the HST observation.

We further confirm the redshift evolution of $\beta$ for the SNLS3 data: for all the IDE models, adding a parameter of $\beta$ can reduce $\chi^{2}$ by $\sim 34$, indicating that $\beta_{1}=0$ is ruled out at $5.8 \sigma \mathrm{CL}$. In addition, we find that the $1 \sigma$ regions of $\beta(z)$ of all these models are almost overlapping, showing that the evolution of $\beta$ is insensitive to the interaction between dark sectors. These results further verify the importance of considering the evolution of $\beta$ in the cosmology fits.

Furthermore, we find that a time-varying $\beta$ has significant effects on the results of parameter estimation: for all the models considered in this paper, varying $\beta$ yields a larger $\Omega_{c 0}$ and a larger $w$; on the other side, varying $\beta$ yields a smaller $h$ for the $w \mathrm{CDM}$ model, while varying $\beta$ has no influence on $h$ for the three IDE models. Moreover, we find that $\gamma$ and $h$ are anti-correlated, showing that there is a degeneracy between $h$ and $\gamma$. In addition, we find that $\gamma$ is tightly constrained in the I $w$ CDM 1 model, but it cannot be well constrained in the $\mathrm{I} w \mathrm{CDM} 2$ and $\mathrm{I} w \mathrm{CDM} 3$ models.

In all, these results show that the evolution of $\beta$ is insensitive to the interaction between dark sectors, and they highlight the importance of considering $\beta$ 's evolution in the cosmology fits.
So far, only the effects of varying $\beta$ on DE models are considered. It is of great interest to study the effects of varying $\beta$ on parameter estimation in MG models. In addition, some other factors, such as the evolution of $\sigma_{\text {int }}$ [155], may also cause the systematic uncertainties of SNe Ia. These issues will be studied in future works.

Acknowledgments We thank the referee for valuable suggestions, which help us to improve this work. We also thank Dr. Daniel Scolnic for helpful discussions. We are grateful to Dr. Alex Conley for providing us with the SNLS3 covariance matrices that allow redshift-dependent $\beta$. We acknowledge the use of CosmoMC. SW is supported by the Fundamental Research Funds for the Central Universities under Grant No. N130305007. XZ is supported by the National Natural Science Foundation of China under Grant No. 11175042 and the Fundamental Research Funds for the Central Universities under Grant No. N120505003.

Open Access This article is distributed under the terms of the Creative Commons Attribution License which permits any use, distribution, and reproduction in any medium, provided the original author(s) and the source are credited.

Funded by $\mathrm{SCOAP}^{3}$ / License Version CC BY 4.0.

\section{References}

1. A.G. Riess et al., Astron. J. 116, 1009 (1998)

2. S. Perlmutter et al., Astrophys. J. 517, 565 (1999)

3. D.N. Spergel et al., Astrophys. J. Suppl. 148, 175 (2003)

4. C.L. Bennet et al., Astrophys. J. Suppl. 148, 1 (2003)

5. D.N. Spergel et al., Astrophys. J. Suppl. 170, 377 (2007)

6. L. Page et al., Astrophys. J. Suppl. 170, 335 (2007)

7. G. Hinshaw et al., Astrophys. J. Suppl. 170, 263 (2007)

8. M. Tegmark et al., Phys. Rev. D 69, 103501 (2004)

9. M. Tegmark et al., Astrophys. J. 606, 702 (2004)

10. M. Tegmark et al., Phys. Rev. D 74, 123507 (2006)

11. E. Komatsu et al., Astrophys. J. Suppl. 180, 330 (2009)

12. E. Komatsu et al., Astrophys. J. Suppl. 192, 18 (2011)

13. W.J. Percival et al., Mon. Not. R. Astron. Soc. 401, 2148 (2010)

14. A.G. Sanchez et al., arXiv:1203.6616 (Mon. Not. R. Astron. Soc. accepted)

15. M. Drinkwater et al., Mon. Not. R. Astron. Soc. 401, 1429 (2010)

16. C. Blake et al., arXiv:1108.2635 (Mon. Not. R. Astron. Soc. accepted)

17. A.G. Riess et al., Astrophys. J. 730, 119 (2011)

18. P.J.E. Peebles, B. Ratra, Astrophys. J. 325, L17 (1988)

19. C. Wetterich, Nucl. Phys. B 302, 668 (1988)

20. R.R. Caldwell, R. Dave, P.J. Steinhardt, Phys. Rev. Lett. 80, 1582 (1998)

21. I. Zlatev, L. Wang, P.J. Steinhardt, Phys. Rev. Lett. 82, 896 (1999)

22. R.R. Caldwell, Phys. Lett. B 545, 23 (2002)

23. S.M. Carroll, M. Hoffman, M. Trodden, Phys. Rev. D 68, 023509 (2003)

24. R.R. Caldwell, M. Kamionkowski, N.N. Weinberg, Phys. Rev. Lett. 91, 071301 (2003)

25. X. Zhang, Eur. Phys. J. C 59, 755 (2009)

26. X. Zhang, Eur. Phys. J. C 60, 661 (2009)

27. X.D. Li et al., Sci. China Phys. Mech. Astron. 55, 1330 (2012)

28. C. Armendariz-Picon, T. Damour, V. Mukhanov, Phys. Lett. B 458, 209 (1999)

29. C. Armendariz-Picon, V. Mukhanov, P.J. Steinhardt, Phys. Rev. D 63, $103510(2001)$ 
30. T. Chiba, T. Okabe, M. Yamaguchi, Phys. Rev. D 62, 023511 (2000)

31. A.Y. Kamenshchik, U. Moschella, V. Pasquier, Phys. Lett. B 511, $265(2001)$

32. M.C. Bento, O. Bertolami, A.A. Sen, Phys. Rev. D 66, 043507 (2002)

33. X. Zhang, F.Q. Wu, J. Zhang, JCAP 01, 003 (2006)

34. K. Liao, Y. Pan, Z.H. Zhu, Res. Astron. Astrophys. 13, 159 (2013)

35. T. Padmanabhan, Phys. Rev. D 66, 021301 (2002)

36. J.S. Bagla, H.K. Jassal, T. Padmanabhan, Phys. Rev. D 67, 063504 (2003)

37. M. Li, Phys. Lett. B 603, 1 (2004)

38. Q.G. Huang, M. Li, JCAP 08, 013 (2004)

39. X. Zhang, F.Q. Wu, Phys. Rev. D 72, 043524 (2005)

40. Z. Chang, F.Q. Wu, X. Zhang, Phys. Lett. B 633, 14 (2006)

41. X. Zhang, F.Q. Wu, Phys. Rev. D 76, 023502 (2007)

42. J.-F. Zhang, X. Zhang, H.-Y. Liu, Eur. Phys. J. C 52, 693 (2007)

43. M. Li, C.S. Lin, Y. Wang, JCAP 05, 023 (2008)

44. M. Li, X.D. Li, S. Wang, X. Zhang, JCAP 06, 036 (2009)

45. X. Zhang, Phys. Lett. B 683, 81 (2010)

46. Y.H. Li, S. Wang, X.D. Li, X. Zhang, JCAP 02, 033 (2013)

47. H. Wei, R.G. Cai, D.F. Zeng, Class. Quantum Gravity 22, 3189 (2005)

48. H. Wei, R.G. Cai, Phys. Rev. D 72, 123507 (2005)

49. H. Wei, N. Tang, S.N. Zhang, Phys. Rev. D 75, 043009 (2007)

50. W. Zhao, Y. Zhang, Class. Quantum Gravity 23, 3405 (2006)

51. T.Y. Xia, Y. Zhang, Phys. Lett. B 656, 19 (2007)

52. S. Wang, Y. Zhang, T.Y. Xia, JCAP 10, 037 (2008)

53. S. Wang, Y. Zhang, Phys. Lett. B 669, 201 (2008)

54. X. Zhang, Phys. Lett. B 648, 1 (2007)

55. X. Zhang, Phys. Rev. D 74, 103505 (2006)

56. J. Zhang, X. Zhang, H. Liu, Phys. Lett. B 651, 84 (2007)

57. J. Zhang, X. Zhang, H. Liu, Eur. Phys. J. C 54, 303 (2008)

58. X. Zhang, Phys. Rev. D 79, 103509 (2009)

59. D. Comelli, M. Pietroni, A. Riotto, Phys. Lett. B 571, 115 (2003)

60. X. Zhang, Mod. Phys. Lett. A 20, 2575 (2005)

61. X. Zhang, Phys. Lett. B 611, 1 (2005)

62. J.A. Frieman, C.T. Hill, A. Stebbins, I. Waga, Phys. Rev. Lett. 75, 2077 (1995)

63. M. Chevallier, D. Polarski, Int. J. Mod. Phys. D 10, 213 (2001)

64. E.V. Linder, Phys. Rev. Lett. 90, 091301 (2003)

65. D. Huterer, G. Starkman, Phys. Rev. Lett. 90, 031301 (2003)

66. D. Huterer, A. Cooray, Phys. Rev. D 71, 023506 (2005)

67. Y. Wang, M. Tegmark, Phys. Rev. Lett. 92, 241302 (2004)

68. Y. Wang, M. Tegmark, Phys. Rev. D 71, 103513 (2005)

69. Y. Wang, K. Freese, Phys. Lett. B 632, 449 (2006)

70. Y. Wang, P. Mukherjee, Astrophys. J. 650, 1 (2006)

71. Y. Wang, P. Mukherjee, Phys. Rev. D 76, 103533 (2007)

72. Y. Wang, Phys. Rev. D 78, 123532 (2008)

73. U. Alam, V. Sahni, T.D. Saini, A.A. Starobinsky, Mon. Not. R. Astron. Soc. 344, 1057 (2003)

74. U. Alam, V. Sahni, T.D. Saini, A.A. Starobinsky, Mon. Not. R. Astron. Soc. 354, 275 (2004)

75. A. Shafieloo, U. Alam, V. Sahni, A.A. Starobinsky, Mon. Not. R. Astron. Soc. 366, 1081 (2006)

76. U. Alam, V. Sahni, A.A. Starobinsky, JCAP 02, 011 (2007)

77. V. Sahni, A. Shafieloo, A.A. Starobinsky, Phys. Rev. D 78, 103502 (2008)

78. A. Shafieloo, V. Sahni, A.A. Starobinsky, Phys. Rev. D 80, 101301(R) (2009)

79. A. Shafieloo, V. Sahni, A.A. Starobinsky, Phys. Rev. D 86, 103527 (2012)

80. J.F. Zhang, X. Zhang, H.Y. Liu, Mod. Phys. Lett. A 23, 139 (2008)

81. Q.G. Huang, M. Li, X.D. Li, S. Wang, Phys. Rev. D 80, 083515 (2009)

82. S. Wang, X.D. Li, M. Li, Phys. Rev. D 82, 103006 (2010)
83. M. Li, X.D. Li, X. Zhang, Sci. China Phys. Mech. Astron. 53, 1631 (2010)

84. S. Wang, X.D. Li, M. Li, Phys. Rev. D 83, 023010 (2011)

85. Y.H. Li, X. Zhang, Eur. Phys. J. C 71, 1700 (2011)

86. X.D. Li et al., JCAP 07, 011 (2011)

87. J.Z. Ma, X. Zhang, Phys. Lett. B 699, 233 (2011)

88. H. Li, X. Zhang, Phys. Lett. B 713, 160 (2012)

89. V. Sahni, S. Habib, Phys. Rev. Lett. 81, 1766 (1998)

90. L. Parker, A. Raval, Phys. Rev. D 60, 063512 (1999)

91. G. Dvali, G. Gabadadze, M. Porrati, Phys. Lett. B 485, 208 (2000)

92. S. Nojiri, S.D. Odintsov, M. Sasaki, Phys. Rev. D 71, 123509 (2005)

93. A. Nicolis, R. Rattazzi, E. Trincherini, Phys. Rev. D 79, 064036 (2009)

94. W. Hu, I. Sawicki, Phys. Rev. D 76, 064004 (2007)

95. A.A. Starobinsky, J. Exp. Theor. Phys. Lett. 86, 157 (2007)

96. G.R. Bengochea, R. Ferraro, Phys. Rev. D 79, 124019 (2009)

97. E.V. Linder, Phys. Rev. D 81, 127301 (2010)

98. T. Harko, F.S.N. Lobo, S. Nojiri, S.D. Odintsov, Phys. Rev. D 84 $024020(2011)$

99. E.J. Copeland, M. Sami, S. Tsujikawa, Int. J. Mod. Phys. D 15, 1753 (2006)

100. J. Frieman, M. Turner, D. Huterer, Ann. Rev. Astron. Astrophys 46, 385 (2008)

101. E.V. Linder, Rept. Prog. Phys. 71, 056901 (2008)

102. R.R. Caldwell, M. Kamionkowski, Ann. Rev. Nucl. Part. Sci. 59, 397 (2009)

103. J.-P. Uzan.arXiv:0908.2243

104. S. Tsujikawa. arXiv: 1004.1493

105. S. Nojiri, S.D. Odintsov, Phys. Rep. 505, 59 (2011)

106. M. Li, X.D. Li, S. Wang, Y. Wang, Commun. Theor. Phys. 56, $525(2011)$

107. T. Clifton, P.G. Ferreira, A. Padilla, C. Skordis, Phys. Rep. 513, 1 (2012)

108. Y. Wang, Dark Energy (Wiley-VCH, New York, 2010)

109. M. Kowalski et al., Astrophys. J. 686, 749 (2008)

110. M. Hicken et al., Astrophys. J. 700, 1097 (2009)

111. M. Hicken et al., Astrophys. J. 700, 331 (2009)

112. R. Amanullah et al., Astrophys. J. 716, 712 (2010)

113. N. Suzuki et al., Astrophys. J. 746, 85 (2012)

114. J. Guy et al., A\&A 523, 7 (2010)

115. A. Conley et al., Astrophys. J. Suppl. 192, 1 (2011)

116. M. Sullivan et al. arXiv: 1104.1444

117. P. Astier et al., Astron. Astrophys. 447, 31 (2006)

118. R. Kessler et al., Astrophys. J. Suppl. 185, 32 (2009)

119. J. Marriner et al. arXiv: 1107.4631

120. D. Scolnic et al. arXiv:1306.4050 (ApJ in press)

121. D. Scolnic et al. arXiv: 1310.3824

122. G. Mohlabeng, J. Ralston. arXiv: 1303.0580

123. S. Wang, Y. Wang, Phys. Rev. D 88, 043511 (2013)

124. S. Wang, Y.H. Li, X. Zhang, Phys. Rev. D 89, 063524 (2014)

125. S. Wang, J.J. Geng, Y.L. Hu, X. Zhang. arXiv: 1312.0184

126. Y. Wang, S. Wang, Phys. Rev. D 88, 043522 (2013)

127. C.H. Chuang, Y. Wang, Mon. Not. R. Astron. Soc. 426, 226 (2012)

128. C.H. Chuang et al. arXiv: 1303.4486

129. G.R. Farrar, P.J.E. Peebles, Astrophys. J. 604, 1 (2004)

130. G. Olivares, F. Atrio-Barandela, D. Pavon, Phys. Rev. D 71, 063523 (2005)

131. T. Koivisto, Phys. Rev. D 72, 043516 (2005)

132. H.M. Sadjadi, M. Alimohammadi, Phys. Rev. D 74, 103007 (2006)

133. Z.K. Guo, N. Ohta, S. Tsujikawa, Phys. Rev. D 76, 023508 (2007)

134. C.G. Boehmer, G. Caldera-Cabral, R. Lazkoz, R. Maartens, Phys. Rev. D 78, 023505 (2008)

135. M. Quartin, M.O. Calvao, S.E. Joras, R.R.R. Reis, I. Waga, JCAP 05, 007 (2008) 
136. J. Valiviita, E. Majerotto, R. Maartens, J. Cosmol. Astropart. Phys. 07, 020 (2008)

137. R. Bean, E.E. Flanagan, I. Laszlo, M. Trodden, Phys. Rev. D 78, 123514 (2008)

138. S. Chongchitnan, Phys. Rev. D 79, 043522 (2009)

139. B.M. Jackson, A. Taylor, A. Berera, Phys. Rev. D 79, 043526 (2009)

140. J. Zhang, H. Liu, X. Zhang, Phys. Lett. B 659, 26 (2008)

141. M. Li, X.-D. Li, S. Wang, Y. Wang, X. Zhang, JCAP 0912, 014 (2009)

142. L. Zhang, J. Cui, J. Zhang, X. Zhang, Int. J. Mod. Phys. D 19, 21 (2010)

143. J. Cui, X. Zhang, Phys. Lett. B 690, 233 (2010)

144. Y. Li, J. Ma, J. Cui, Z. Wang, X. Zhang, Sci. China Phys. Mech. Astron. 54, 1367 (2011)

145. T.-F. Fu, J.-F. Zhang, J.-Q. Chen, X. Zhang, Eur. Phys. J. C 72, $1932(2012)$

146. Z. Zhang, S. Li, X.-D. Li, X. Zhang, M. Li, JCAP 1206, 009 (2012)

147. T. Clemson, K. Koyama, G.B. Zhao, R. Maartens, J. Valiviita, Phys. Rev. D 85, 043007 (2012)
148. J. Zhang, L. Zhao, X. Zhang, Sci. China Phys. Mech. Astron. 57, 387 (2014)

149. J.H. He, B. Wang, JCAP 06, 010 (2008)

150. J.H. He, B. Wang, P. Zhang, Phys. Rev. D 80, 063530 (2009)

151. J.H. He, B. Wang, E. Abdalla, D. Pavon, JCAP 12, 022 (2010)

152. X.D. Xu, B. Wang, E. Abdalla, Phys. Rev. D 85, 083513 (2012)

153. X.D. Xu, B. Wang, P. Zhang, F. Atrio-Barandela, JCAP 12, 001 (2013)

154. Y.H. Li, X. Zhang, Phys. Rev. D 89, 083009 (2014)

155. A. Kim. arXiv: 1101.3513

156. Y.H. Li, J.F. Zhang, X. Zhang, Phys. Rev. D 90, 063005 (2014)

157. A. Lewis, S. Bridle, Phys. Rev. D 66, 103511 (2002)

158. J.J. Geng, J.F. Zhang, X. Zhang, JCAP 07, 006 (2014) 